\title{
Error Bounds for Linear Recurrence Relations*
}

\author{
By F. W. J. Olver
}

\begin{abstract}
Recurrence relations of the form

$$
a_{r} p_{r+1}=b_{r} p_{r}+c_{r} p_{r-1}
$$

are examined in two cases: (A) oscillatory systems, for which $b_{r}^{2}+4 a_{r} c_{r}<0$; (B) monotonic systems, for which $b_{r}^{2}+4 a_{r} c_{r} \geq 0$. In both cases, a posteriori methods are supplied for constructing strict and realistic error bounds in $O(r)$ arithmetic operations. A priori bounds, also requiring $O(r)$ arithmetic operations, are supplied in Case B. Several illustrative numerical examples are included.
\end{abstract}

1. Introduction. The application of $m$ th order linear recurrence relations

$$
a_{r 0} p_{r}+a_{r 1} p_{r-1}+a_{r 2} p_{r-2}+\cdots+a_{r m} p_{r-m}+d_{r}=0,
$$

in which $a_{r 0} \neq 0$, all $r$, to generate a sequence of values $p_{m}, p_{m+1}, \ldots$ from prescribed values of $p_{0}, p_{1}, \ldots, p_{m-1}$ is a well-understood procedure in numerical analysis. See, for example, [1], [2], [3], [4] and, most recently, the monograph of Wimp [19]. If the corresponding homogeneous equation is regarded as a difference equation, then it has $m$ linearly independent solutions - the so-called complementary functions of (1.1). Each rounding error introduced in the recurrence process contaminates the wanted solution of $(1.1)$ by small multiples of the complementary functions. This is of no concern if the wanted solution grows in size at least as fast as any of the complementary functions, that is, if it is a dominant solution. In other cases the process may fail, indeed fail disastrously, and in order to achieve stability it is necessary to apply the recurrence relation in a backward direction, or to solve a boundary value problem.

Perhaps because stability conditions are so well understood, comparatively little attention has been paid to the problem of constructing strict error bounds for the computed results. These bounds are to cover the effects of rounding errors introduced during the recurrence steps as well as inherent errors in the coefficients $a_{r j}$ and $d_{r}$ and the initial values $p_{0}, p_{1}, \ldots, p_{m-1}$. This is the problem treated in the present investigation. One obvious application is to the development of robust software for the generation of transcendental mathematical functions by recurrence.

The only relevant published work appears to be that for Miller's algorithm; see [7], [9], [16]. In fact, some results for the present problem could be found simply by specializing results given in these references, especially [7]. This approach leads to unnecessary complications, however, and a more direct attack is called for.

Received October 30, 1986, revised July 6, 1987.

1980 Mathematics Subject Classification (1985 Revision). Primary 65Q05; Secondary 65G05.

*This research has been supported by the U. S. Army Research Office, Durham under Contract DAAG-29-84-K-0022, the U. S. National Science Foundation under Grant DMS-84-19820 and the U. K. Science and Engineering Research Council. 
We first observe that the evaluation of $p_{r}$ for the range $r=m, m+1, \ldots, m+n-1$, say, is equivalent to the solution of a system of $n$ linear algebraic equations. Hence the required error bounds can be found by available algorithms in matrix algebra; see, for example, [13], [14]. A drawback to this approach is that it requires the inversion of a lower triangular band matrix. The number of arithmetic operations needed for the inversion is $O\left(n^{2}\right)$, for large $n$, compared with only $O(n)$ operations for the computation of the solution $p_{r}$. It can be argued that it suffices to have the norm of the inverse matrix. However, it is an upper bound for the norm that is really needed, and this is tantamount to the original problem. ${ }^{1}$

Another drawback to the matrix approach is that it usually fails to provide insight into the nature of the error bounds; in particular, it will not yield realistic bounds of a priori type unless, of course, bounds for the elements or norm of the inverse matrix are known.

A second general approach is to apply rounded interval arithmetic [8, Section 2.4]. Often this procedure is quite successful. In many cases, however, the computed intervals are absurdly unrealistic. We illustrate this observation by two simple examples.

Example 1.1.

$$
12 p_{r+1}=25 p_{r}-13 p_{r-1} ; \quad p_{0}=1, p_{1}=13 / 12
$$

Computed interval values of $p_{2}, p_{3}, \ldots, p_{16}$ are given in Table 1.1. For example, the entries for $r=2$ mean that

$$
1.17360 \leq p_{2} \leq 1.17363
$$

Six-figure decimal arithmetic was employed, with directed rounding ${ }^{2}$ applied immediately following each arithmetic operation at each recurrence step.

Clearly the interval widths grow rapidly as $r$ increases. After $r=12$ the left endpoint begins to decrease and actually becomes negative at $r=16$, even though the true solution $p_{r}=(13 / 12)^{r}$ is positive, increasing and dominant.

Example 1.2.

$$
3 p_{r+1}-\sqrt{22} p_{r}+2 p_{r-1}-1=0 ; \quad p_{0}=p_{1}=1
$$

An interval solution was computed in the same manner as Example 1.1, and the results are presented in Table 1.2. Again the interval widths grow rapidly with $r$, even though the wanted solution is dominant and tends to the constant value $3.23013 \ldots$ as $r \rightarrow \infty$. The actual solution is given by

$$
p_{r}=\frac{1}{3}(5+\sqrt{22})-2^{r / 2} 3^{-(r+2) / 2}\{(2+\sqrt{22}) \cos r \omega+(\sqrt{176}-\sqrt{50}) \sin r \omega\},
$$

with $\omega=\tan ^{-1}(1 / \sqrt{11})$.

\footnotetext{
${ }^{1}$ Compare [5]. Here algorithms are supplied for computing the norm of the inverse of a tridiagonal matrix of order $n$ in $O(n)$ operations. The algorithms entail the application of three-term homogeneous recurrence relations.

${ }^{2}$ That is, towards $-\infty$ for left endpoints and towards $+\infty$ for right endpoints.
} 
TABLE 1.1

Interval solution of (1.2)

\begin{tabular}{|c|c|}
\hline 1 & 1 \\
\hline 1.08333 & 1.08334 \\
\hline 1.17360 & 1.17363 \\
\hline 1.27137 & 1.27147 \\
\hline 1.37725 & 1.37750 \\
\hline 1.49183 & 1.49248 \\
\hline 1.61568 & 1.61732 \\
\hline 1.74914 & 1.75328 \\
\hline 1.89194 & 1.90235 \\
\hline 2.04215 & 2.06834 \\
\hline 2.19359 & 2.25945 \\
\hline 2.32926 & 2.49487 \\
\hline 2.40488 & 2.82127 \\
\hline 2.30738 & 3.35430 \\
\hline 1.75065 & 4.38285 \\
\hline 0.0133583 & 6.63135 \\
\hline-4.72027 & 11.9189 \\
\hline
\end{tabular}

TABLE 1.2

Interval solution of (1.3)

\begin{tabular}{l|r}
$I p_{r+1} / I p_{r}$ & $r$ \\
\multicolumn{1}{c}{$\ldots$} & 0 \\
3.0 & 1 \\
$3.333 \ldots$ & 2 \\
2.5 & 3 \\
2.6 & 4 \\
$2.523 \ldots$ & 5 \\
$2.524 \ldots$ & 6 \\
$2.514 \ldots$ & 7 \\
$2.515 \ldots$ & 8 \\
$2.514 \ldots$ & 9 \\
$2.514 \ldots$ & 10 \\
$2.514 \ldots$ & 11 \\
$2.514 \ldots$ & 12 \\
$2.514 \ldots$ & 13 \\
$2.514 \ldots$ & 14 \\
$2.514 \ldots$ & 15 \\
& 16
\end{tabular}

\begin{tabular}{|c|c|c|}
\hline & & $I p_{r+1} / I p_{r}$ \\
\hline 1 & 1 & - \\
\hline 1 & 1 & - \\
\hline 1.23013 & 1.23014 & 3.0 \\
\hline 1.58993 & 1.58996 & 2.333 . \\
\hline 1.99904 & 1.99911 & 2.142 . \\
\hline 2.39879 & 2.39894 & 2.133 . \\
\hline 2.75102 & 2.75134 & 2.0 \\
\hline 3.03517 & 3.03581 & $2.015 \ldots$ \\
\hline 3.24447 & 3.24576 & $1.968 \ldots$ \\
\hline 3.38203 & 3.38457 & $1.933 \ldots$ \\
\hline 3.45716 & 3.46207 & 1.930 . \\
\hline 3.48206 & 3.49154 & 1.916 \\
\hline 3.46933 & 3.48750 & 1.915. \\
\hline 3.42980 & 3.46460 & $1.913 \ldots$ \\
\hline 3.37070 & 3.43730 & $1.912 \ldots$ \\
\hline 3.29356 & 3.42094 & $1.912 .$. \\
\hline 3.19116 & 3.43477 & \\
\hline
\end{tabular}

The explanation of the failure of interval arithmetic in these examples is the usual one: the process takes no account of the interdependence of errors at successive steps. In fact, in Example 1.1 the interval widths $I p_{r}$, say, eventually grow in proportion to $\alpha^{r}$, where $\alpha=2.514 \ldots$ is the largest zero of the polynomial $12 z^{2}-$ $25 z-13$. This is confirmed by the numerical values of the ratio $I p_{r+1} / I p_{r}$ given in the final column of Table 1.1. Similarly in Example 1.2 the interval widths eventually grow in proportion to $\alpha^{r}$, where $\alpha=1.912 \ldots$ is the largest zero of $3 z^{2}-\sqrt{22} z-2$.

To construct methods that entail no more than $O(r)$ arithmetic operations and yield realistic error bounds, we have to impose restrictions on the nature of the recurrence relation. Without such restrictions, we have only the general matrix approach, with its $O\left(r^{2}\right)$ operations, to fall back on for realistic bounds. The present paper treats only real second-order relations. We also restrict ourselves to homogeneous systems, mainly because inhomogeneous problems often require error bounds for the associated complementary functions as a necessary preliminary $[1],[10],[19]$. In some cases, however, our methods carry over straightforwardly to inhomogeneous systems. Admittedly, the problems that fall within our scope amount to only a small subclass of the general problem of solving linear difference equations; nevertheless, this subclass includes many important recurrence relations satisfied by the higher transcendental functions.

We standardize (1.1) for homogeneous second-order systems in the form

$$
a_{r} p_{r+1}=b_{r} p_{r}+c_{r} p_{r-1}
$$

with $p_{0}$ and $p_{1}$ prescribed and $a_{r} \neq 0$, all $r$. We distinguish two cases: oscillatory systems in which $b_{r}^{2}+4 a_{r} c_{r}$ is negative for all $r$, and monotonic systems in which $b_{r}^{2}+4 a_{r} c_{r}$ is nonnegative for all $r$. This classification is suggested, of course, by the nature of the solutions when the $a_{r}, b_{r}$ and $c_{r}$ are constants. Oscillatory systems are treated in Section 2, and monotonic systems in Sections 3, 4 and 5. In both cases we provide methods for constructing error bounds of a posteriori type. For 
monotonic systems we also furnish a priori bounds. Some numerical examples are supplied in Section 6, and brief conclusions are drawn in Section 7.

2. Oscillatory Systems. In (1.4) we replace $c_{r}$ by $-c_{r}$ for convenience. The oscillatory case is then given by

$$
a_{r} p_{r+1}=b_{r} p_{r}-c_{r} p_{r-1},
$$

with $b_{r}^{2}<4 a_{r} c_{r}$, all $r$. Without loss of generality we may suppose that $a_{r}$ and $c_{r}$ are positive.

Example 1.2 is typical for systems of this kind in that interval arithmetic will generally yield unsatisfactory results. The error bounds, or interval widths, eventually grow at the same rate as the dominant solution of the equation

$$
a_{r} p_{r+1}=\left|b_{r}\right| p_{r}+c_{r} p_{r-1} .
$$

That this solution grows faster than the solutions of $(2.1)$ can be inferred from the case in which the coefficients are constants.

In order to proceed, let $q_{r}$ be a solution of (2.1) that is independent of $p_{r}$ and (like $p_{r}$ ) is computed by forward recurrence from given values at $r=0$ and 1 . Denote the stored values of $p_{r}, q_{r}$ and other quantities by the addition of overbars. Also, let $\phi_{r}$ and $\psi_{r}$ be the aggregate errors introduced on the $(r-1)$ st step in the computation of $p_{r}$ and $q_{r}$, as expressed by the formulae

$$
a_{r-1} \bar{p}_{r}=b_{r-1} \bar{p}_{r-1}-c_{r-1} \bar{p}_{r-2}+\phi_{r}, \quad a_{r-1} \bar{q}_{r}=b_{r-1} \bar{q}_{r-1}-c_{r-1} \bar{q}_{r-2}+\psi_{r} .
$$

Thus $\phi_{r}$ includes the effects of all abbreviation errors ${ }^{3}$ introduced in the computation of $\bar{p}_{r}$ from $\bar{p}_{r-1}$ and $\bar{p}_{r-2}$ as well as the effects of inherent errors in the given values of the coefficients $a_{r-1}, b_{r-1}$ and $c_{r-1}$. Similarly for $\psi_{r}$.

Bounds for $\left|\phi_{r}\right|$ and $\left|\psi_{r}\right|$ can be computed by standard methods of round-off error analysis, see for example [12], [18], or by interval arithmetic. For the initial values we set

$$
\bar{p}_{0}=p_{0}+\phi_{0}, \quad \bar{p}_{1}=p_{1}+\phi_{1}, \quad \bar{q}_{0}=q_{0}+\psi_{0}, \quad \bar{q}_{1}=q_{1}+\psi_{1} .
$$

The relationship of the stored values $\bar{p}_{r}$ and $\bar{q}_{r}$ to the true values $p_{r}$ and $q_{r}$ is easily verified to be

$$
\bar{p}_{r}=p_{r}+B_{r} p_{r}-A_{r} q_{r}, \quad \bar{q}_{r}=q_{r}+D_{r} p_{r}-C_{r} q_{r},
$$

where

$$
\begin{array}{ll}
A_{r}=-w_{1} p_{1} \phi_{0}+\sum_{j=1}^{r} w_{j} p_{j-1} \phi_{j}, & B_{r}=-w_{1} q_{1} \phi_{0}+\sum_{j=1}^{r} w_{j} q_{j-1} \phi_{j} \\
C_{r}=-w_{1} p_{1} \psi_{0}+\sum_{j=1}^{r} w_{j} p_{j-1} \psi_{j}, & D_{r}=-w_{1} q_{1} \psi_{0}+\sum_{j=1}^{r} w_{j} q_{j-1} \psi_{j}
\end{array}
$$

and

$$
w_{1}=\frac{1}{p_{1} q_{0}-p_{0} q_{1}}, \quad w_{r}=\frac{1}{a_{r-1}\left(p_{r} q_{r-1}-p_{r-1} q_{r}\right)}, \quad r \geq 2 .
$$

\footnotetext{
${ }^{3} \mathrm{By}$ "abbreviation errors" we mean chopping or rounding errors.
} 
The $w_{r}$ are finite since $p_{r}$ and $q_{r}$ are assumed to be independent solutions. We also have the recurrence relation

$$
w_{r}=\left(a_{r-2} / c_{r-1}\right) w_{r-1}, \quad r \geq 3,
$$

and $w_{2}=w_{1} / c_{1}$. Let us denote the wanted errors by

$$
\varepsilon_{r}=p_{r}-\bar{p}_{r}, \quad \eta_{r}=q_{r}-\bar{q}_{r} .
$$

Suppose that we have computed $\bar{p}_{r}$ and $\bar{q}_{r}$, together with bounds on $\left|p_{j}\right|,\left|q_{j}\right|$, $\left|\varepsilon_{j}\right|,\left|\eta_{j}\right|,\left|A_{j}\right|,\left|B_{j}\right|,\left|C_{j}\right|,\left|D_{j}\right|$ and $\left|w_{j}\right|$, for all $j \leq r-1$. We first compute bounds on $\left|\phi_{r}\right|,\left|\psi_{r}\right|$ and $\left|w_{r}\right|$; compare (2.2) and (2.8). Next, from (2.5) and (2.6) we have

$$
\begin{array}{ll}
A_{r}=A_{r-1}+w_{r} p_{r-1} \phi_{r}, & B_{r}=B_{r-1}+w_{r} q_{r-1} \phi_{r}, \\
C_{r}=C_{r-1}+w_{r} p_{r-1} \psi_{r}, & D_{r}=D_{r-1}+w_{r} q_{r-1} \psi_{r},
\end{array}
$$

provided that $r \geq 1$. Using these relations we compute bounds on $\left|A_{r}\right|,\left|B_{r}\right|,\left|C_{r}\right|$ and $\left|D_{r}\right|$. Then by substituting the results obtained so far into the identities

$$
\begin{aligned}
& E_{r} \varepsilon_{r}=-\left\{B_{r}\left(1-C_{r}\right)+A_{r} D_{r}\right\} \bar{p}_{r}+A_{r} \bar{q}_{r} \\
& E_{r} \eta_{r}=-D_{r} \bar{p}_{r}+\left\{\left(1+B_{r}\right) C_{r}-A_{r} D_{r}\right\} \bar{q}_{r}
\end{aligned}
$$

in which

$$
E_{r}=\left(1+B_{r}\right)\left(1-C_{r}\right)+A_{r} D_{r}
$$

we arrive at bounds for $\left|\varepsilon_{r}\right|$ and $\left|\eta_{r}\right|$. (These identities are obtained by solving Eqs. (2.4) for $p_{r}$ and $q_{r}$, and using (2.9).) Bounds for $p_{r}$ and $q_{r}$ follow from (2.9), and after computing $\bar{p}_{r+1}$ and $\bar{q}_{r+1}$ from (2.1) we are ready to repeat the cycle.

This is our method for constructing a posteriori error bounds. The magnitudes of the solutions $p_{r}$ and $q_{r}$ may rise or fall as $r$ increases, depending on whether $c_{r} \gtrless a_{r}$. However, provided that the rate of growth of the magnitudes of the solutions does not differ significantly from that of $\left(c_{r} / a_{r}\right)^{1 / 2}$, all terms in the sums in $(2.5)$ and (2.6) will remain of comparable magnitude, owing to the presence of the factors $w_{j}$. That this growth condition is not unreasonable can be seen by analogy with the case in which the difference equation has constant coefficients. Nevertheless, the condition will not always be satisfied in the general case, and it may need to be examined by asymptotic analysis or other independent means.

When the growth condition just discussed is satisfied, the bounds for $\left|A_{r}\right|,\left|B_{r}\right|$, $\left|C_{r}\right|$ and $\left|D_{r}\right|$ may be expected to grow approximately linearly with $r$, which is an essential requirement for the bounds for $\left|\varepsilon_{r}\right|$ and $\left|\eta_{r}\right|$ to be realistic. The number of arithmetic operations needed is several times that required to compute the $\bar{p}_{r}$, of course, but is still only $O(r)$ for large $r$. Moreover, many of these computations could be performed in parallel: if this is arranged, then the total execution time will not greatly exceed that needed for the computation of the $\bar{p}_{r}$ alone. Lastly, the method can be extended easily to inhomogeneous oscillatory systems, as long as the wanted solution is not dominated by the complementary functions as $r$ increases.

3. Monotonic Systems (i). We now consider Eq. (1.4), that is,

$$
a_{r} p_{r+1}=b_{r} p_{r}+c_{r} p_{r-1}
$$


with the condition $b_{r}^{2}+4 a_{r} c_{r} \geq 0$, all $r$. We may suppose that $a_{r}>0$, and we shall also suppose that $b_{r} \geq 0 .{ }^{4}$ In the present section we require $c_{r} \geq 0$, deferring the more difficult case of negative $c_{r}$ until Sections 4 and 5 .

The essential behavior in this case is that for appropriately chosen solutions the relative errors are simply additive. To express this result precisely and conveniently, we use relative precision ( $\mathrm{rp}$ ) in place of relative error, that is, we work in terms of the absolute errors of the logarithms of approximations [12].

We assume that the stored values $\bar{a}_{r}, \bar{b}_{r}$ and $\bar{c}_{r}$ of $a_{r}, b_{r}$ and $c_{r}$, respectively, are correct to $\operatorname{rp}(\delta)$, say, and the computations are performed in floating-point arithmetic with a working relative precision (wrp) of $\gamma$. (In other words, each arithmetic operation is accompanied by a chopping or rounding error not exceeding $\operatorname{rp}(\gamma)$.) We also assume that the initial values satisfy

$$
p_{0} \simeq \bar{p}_{0} ; \quad \operatorname{rp}(\varpi), \quad p_{1} \simeq \bar{p}_{1} ; \quad \operatorname{rp}(\varpi),
$$

where $\bar{p}_{0}$ and $\bar{p}_{1}$ are nonnegative, and $\varpi$, like $\delta$ and $\gamma$, is given. (Without these assumptions, $p_{r}$ might be recessive as $r \rightarrow \infty$.) By application of the rules of $\mathrm{rp}$ error analysis and a simple inductive argument we deduce that

$$
p_{r} \simeq \bar{p}_{r} ; \quad \operatorname{rp}\{\varpi+(2 r-2) \delta+(3 r-3) \gamma\}, \quad r \geq 1 .
$$

This is the required result. Often it is improvable in minor ways. For example, if $a_{r}=1$, all $r$, then the coefficients of $\delta$ and $\gamma$ can be reduced to $r-1$ and $2 r-2$, respectively.

It should also be noted that if interval arithmetic is applied directly to (3.1), then it will yield realistic a posteriori bounds. However, in view of the simplicity and effectiveness of the a priori bounds just given, the extra computations entailed by use of interval arithmetic can be avoided.

4. Monotonic Systems (ii). In this and the next section we consider the equation

$$
a_{r} p_{r+1}=b_{r} p_{r}-c_{r} p_{r-1}
$$

in which $b_{r}^{2} \geq 4 a_{r} c_{r}, a_{r}>0, b_{r}>0$ and $c_{r} \geq 0$, for all $r$. We seek a solution $p_{r}$ such that $p_{r} \geq 0$, for all $r$.

For reasons similar to those given in the oscillatory case (Section 2), interval arithmetic applied directly to (4.1) will yield unsatisfactory results. The method of Section 2 also fails. If $p_{r}$ is dominant and $q_{r}$ is recessive as $r \rightarrow \infty$, then in the second of (2.4) the term $D_{r} p_{r}$ soon overwhelms $q_{r}$. If $p_{r}$ and $q_{r}$ are both dominant, then the situation is even worse.

One way to proceed is to transform (4.1) into the nonlinear equation

$$
a_{r} h_{r+1}=b_{r}-\left(c_{r} / h_{r}\right)
$$

satisfied by the ratio $h_{r}=p_{r} / p_{r-1}$. Then interval arithmetic, or a running error analysis [12], [18], can be applied to the computation of the sequence $\left\{h_{r}\right\}$ by recurrence, and also to the subsequent recovery of the wanted solution from the product

$$
p_{r}=h_{r} h_{r-1} \cdots h_{1} p_{0} .
$$

4 Systems in which $a_{r}$ and $b_{r}$ have opposite signs for all $r$ are accommodated by replacing $p_{r}$ by $(-1)^{r} p_{r}$. 
The reason these procedures are now more successful is that they make appropriate allowance for interactions of errors. In contrast, when (4.1) is computed in interval form, the upper (say) endpoint of $p_{r+1}$ depends on the upper endpoint of $p_{r}$ and the lower endpoint of $p_{r-1}$.

Another approach is to replace (4.1) by a pair of first-order linear equations with nonnegative coefficients; compare Section 3. For example, we can introduce a new variable $u_{r}$ defined by

$$
u_{r}=p_{r+1}-\lambda_{r} p_{r}
$$

where $\lambda_{r}$ is a positive function of $r$ at our disposal, subject to the condition $u_{r} \geq 0$. Then (4.1) is equivalent to

$$
a_{r} u_{r}=\nu_{r} p_{r}+\mu_{r-1} u_{r-1}, \quad p_{r+1}=\lambda_{r} p_{r}+u_{r}
$$

where

$$
\mu_{r-1}=c_{r} / \lambda_{r-1}, \quad \nu_{r}=b_{r}-a_{r} \lambda_{r}-\mu_{r-1} .
$$

By hypothesis, $\lambda_{r-1}>0$, hence $\mu_{r-1}$ is finite and nonnegative. The remaining coefficient $\nu_{r}$ is nonnegative as long as $\lambda_{r}$ and $\lambda_{r-1}$ also satisfy

$$
a_{r} \lambda_{r-1} \lambda_{r}-b_{r} \lambda_{r-1}+c_{r} \leq 0 .
$$

If the coefficients $a_{r}, b_{r}$ and $c_{r}$ are slowly-varying functions of $r$ such that $b_{r}^{2}>$ $4 a_{r} c_{r}$ and the starting values $p_{0}, p_{1}$ are chosen appropriately, then it will usually be possible to satisfy (4.6). This is because the zeros of the local characteristic polynomial $a_{r} z^{2}-b_{r} z+c_{r}$ are real and distinct, and in effect (4.6) requires $\lambda_{r-1}$ and $\lambda_{r}$ to lie between them. For example, we might choose $\lambda_{r}$ to be the arithmetic mean of the zeros, given by

$$
\lambda_{r}=b_{r} /\left(2 a_{r}\right) .
$$

Then (4.6) is satisfied as long as

$$
b_{r-1} b_{r} \geq 4 a_{r-1} c_{r}, \quad \text { all } r .
$$

Solutions of (4.4) may be generated by interval arithmetic or with a running error analysis. Considerable cancellation may occur in the computation of $\nu_{r}$ from the second of (4.5); in consequence, it may be necessary to employ higher precision on this step.

In the next section we describe a semianalytical method. This method provides greater insight into the actual error propagation, and leads to useful a priori bounds. It has some features in common with the valuable method used by Mattheij and van der Sluis for obtaining error bounds for Miller's algorithm [7].

5. Monotonic Systems (iii). As in Section 4 we consider the equation

$$
a_{r} p_{r+1}=b_{r} p_{r}-c_{r} p_{r-1},
$$

but with the conditions on the coefficients modified to $b_{r}^{2}>4 a_{r} c_{r}, a_{r}>0, b_{r}>0$ and $c_{r}>0$, for all $r$. Again, we wish to compute a solution $p_{r}$ that is dominant as $r \rightarrow \infty$. We suppose that $p_{r}$ is positive when $r>0$ and nonnegative when $r=0$. To begin with, we denote by $q_{r}$ any positive solution that is independent of $p_{r}$. 
As in earlier sections, we use overbars to indicate stored values. We first investigate the actual propagation of the aggregate abbreviation error $\phi_{j}$, say, introduced on the $(j-1)$ st application of $(5.1)$ according to the formula

$$
a_{j-1} \bar{p}_{j}=b_{j-1} \bar{p}_{j-1}-c_{j-1} \bar{p}_{j-2}+\phi_{j}, \quad j \geq 2
$$

compare (2.2). The solution $p_{r}^{(j)}$, say, of (5.1) that satisfies

$$
p_{j-1}^{(j)}=0, \quad p_{j}^{(j)}=\phi_{j} / a_{j-1}, \quad j \geq 2,
$$

is expressible in the form

$$
p_{r}^{(j)}=\left(1-\frac{p_{j-1} q_{r}}{q_{j-1} p_{r}}\right) \frac{t_{j} \phi_{j}}{a_{j-1} p_{j}} p_{r}
$$

where

$$
t_{j}=\left(1-\frac{p_{j-1} q_{j}}{q_{j-1} p_{j}}\right)^{-1}, \quad j \geq 1 .
$$

With the assumed conditions, $t_{j}$ is always finite.

Now suppose that $q_{r}$ is the recessive solution of $(5.1)$, so that $q_{r} / p_{r} \rightarrow 0$ as $r \rightarrow \infty$. Although $q_{r}$ is unique only up to a constant factor, obviously from (5.4) the coefficients $t_{j}$ in (5.3) do not depend on this factor. Furthermore, from (5.3) we have

$$
\frac{p_{r}^{(j)}}{p_{r}} \rightarrow \frac{t_{j} \phi_{j}}{a_{j-1} p_{j}}, \quad r \rightarrow \infty, \quad j \text { fixed. }
$$

This means that the relative error $\phi_{j} /\left(a_{j-1} p_{j}\right)$ introduced on the $(j-1)$ st application of $(5.1)$ is magnified ultimately by the factor $t_{j}$. If it happens that $q_{r} / p_{r}$ is decreasing for all $r$, then we have, in addition,

$$
\frac{\left|p_{r}^{(j)}\right|}{p_{r}} \leq \frac{t_{j}\left|\phi_{j}\right|}{a_{j-1} p_{j}}, \quad r \geq j .
$$

In other words, the actual propagated error is bounded by its limiting form. It also has the same sign.

For our purposes, it is not essential for $q_{r}$ to be the recessive solution. Suppose that we are computing $p_{r}$ over the range $r=2,3, \ldots, n$, where $n$ is arbitrary. Let $q_{r}$ now denote any solution of (5.1) that is positive when $0 \leq r \leq n-1$, nonnegative when $r=n$ and also has the property that $q_{r} / p_{r}$ is decreasing for $0 \leq r \leq n$. Then $q_{r}$ is independent of $p_{r}$; furthermore, if $t_{j}$ is defined by (5.4) in terms of the present $q_{r}$, then (5.6) applies for $j=2,3, \ldots, n$.

To investigate the effect of inherent errors in the starting values at $r=0$ and 1 , let

$$
p_{0}=\bar{p}_{0}-\phi_{0}, \quad p_{1}=\bar{p}_{1}-\phi_{1},
$$

as in (2.3). Then the solution $p_{r}^{(0)}$, say, of (5.1) that satisfies

$$
p_{0}^{(0)}=-\phi_{0}, \quad p_{1}^{(0)}=-\phi_{1},
$$

is given by

$$
p_{r}^{(0)}=\left(1-\frac{p_{1} q_{r}}{q_{1} p_{r}}\right) \frac{t_{0} \phi_{0}}{p_{0}} p_{r}-\left(1-\frac{p_{0} q_{r}}{q_{0} p_{r}}\right) \frac{t_{1} \phi_{1}}{p_{1}} p_{r}
$$


where $t_{1}$ is defined as in (5.4) and

$$
t_{0}=\left(\frac{q_{0} p_{1}}{p_{0} q_{1}}-1\right)^{-1} .
$$

With the assumed conditions we have

$$
\frac{\left|p_{r}^{(0)}\right|}{p_{r}} \leq \frac{t_{0}\left|\phi_{0}\right|}{p_{0}}+\frac{t_{1}\left|\phi_{1}\right|}{p_{1}}, \quad r \geq 1
$$

On combining the effects of all the errors $\phi_{0}, \phi_{1}, \ldots, \phi_{r}$ we arrive at

$$
\frac{\left|p_{r}-\bar{p}_{r}\right|}{p_{r}} \leq t_{0} \frac{\left|\phi_{0}\right|}{p_{0}}+t_{1} \frac{\left|\phi_{1}\right|}{p_{1}}+\sum_{j=2}^{r} t_{j} \frac{\left|\phi_{j}\right|}{a_{j-1} p_{j}}, \quad 2 \leq r \leq n .
$$

In the relations $(5.9)$ to $(5.12)$ we have supposed that $p_{0} \neq 0$. If $p_{0}=0$, then we suppose that $\bar{p}_{0}=0$. The inequalities (5.11) and (5.12) then apply without the term $t_{0}\left|\phi_{0}\right| / p_{0}$ on their right-hand sides. ${ }^{5}$

In order to proceed, we need bounds on the coefficients $t_{j}$ defined by (5.4) and (5.10). In turn, this necessitates bounds on $p_{j-1} / p_{j}$ and $q_{j} / q_{j-1}$. Results of this kind have been supplied by the present writer [11], Mattheij [6] and van der Sluis [15]. For present purposes a simple and convenient result is provided by the following theorem. This result is included in that given by Theorem 4.1 of [6], but for simplicity we give a proof using our present notation.

THEOREM 5.1. Let $\alpha_{r}$ and $\beta_{r}$ denote the (positive) zeros of the quadratic $a_{r} z^{2}$ $-b_{r} z+c_{r}$, chosen so that $\alpha_{r}>\beta_{r}$. Write

$$
\begin{gathered}
\alpha=\min \left(\alpha_{1}, \alpha_{2}, \ldots, \alpha_{n-1}\right), \quad \mathrm{A}=\max \left(\alpha_{1}, \alpha_{2}, \ldots, \alpha_{n-1}\right), \\
\mathrm{B}=\max \left(\beta_{1}, \beta_{2}, \ldots, \beta_{n-1}\right),
\end{gathered}
$$

and assume that $\alpha \geq \mathrm{B}$. Also, let $v_{r}$ be any solution of (5.1) that is nonnegative when $r=0$ and satisfies $v_{1} / v_{0} \geq \mathrm{B}$. Then

$$
\hat{\alpha} \leq v_{r} / v_{r-1} \leq \hat{\mathrm{A}}, \quad r=1,2, \ldots, n,
$$

where

$$
\hat{\alpha}=\min \left(\alpha, v_{1} / v_{0}\right), \quad \hat{\mathrm{A}}=\max \left(\mathrm{A}, v_{1} / v_{0}\right) .
$$

(In the case $v_{0}=0$ the condition $v_{1} / v_{0} \geq \mathrm{B}$ becomes $v_{1}>0, \hat{\alpha}=\alpha$ and $\hat{\mathrm{A}}=\infty$.)

To prove the theorem, write

$$
f_{r}(z)=\frac{b_{r}}{a_{r}}-\frac{c_{r}}{a_{r} z}
$$

so that

$$
v_{r+1} / v_{r}=f_{r}\left(v_{r} / v_{r-1}\right) .
$$

We observe that for fixed $r, f_{r}(z)$ is increasing when $z>0$ and

$$
f_{r}(z) \leq z, \quad \text { if } z \geq \alpha_{r} ; \quad f_{r}(z) \geq z, \quad \text { if } \beta_{r} \leq z \leq \alpha_{r} .
$$

\footnotetext{
${ }^{5}$ An appropriate modification could be made, however, if $p_{0}=0$ but $\bar{p}_{0} \neq 0$.
} 
From these results and the identity $f_{r}\left(\alpha_{r}\right)=\alpha_{r}$ it follows that:
(a) if $\alpha_{r} \leq v_{r} / v_{r-1}$
then $\alpha_{r} \leq v_{r+1} / v_{r} \leq v_{r} / v_{r-1}$;
(b) if $\beta_{r} \leq v_{r} / v_{r-1} \leq \alpha_{r}$,
then $v_{r} / v_{r-1} \leq v_{r+1} / v_{r} \leq \alpha_{r}$.

The result (5.14) is now proved by induction. Suppose that $v_{r} / v_{r-1} \geq \mathrm{B}$ and $\hat{\alpha} \leq v_{r} / v_{r-1} \leq \hat{\mathrm{A}}$, as is certainly the case when $r=1$. Then $v_{r} / v_{r-1} \geq \beta_{r}$. Hence (a) or (b) applies. In either event we have $v_{r+1} / v_{r} \geq \mathrm{B}$ and $\hat{\alpha} \leq v_{r+1} / v_{r} \leq \hat{\mathrm{A}}$.

Let us return to the bound (5.12). Defining $\alpha$ and B by (5.13) and applying Theorem 5.1, we find that

$$
p_{r-1} / p_{r} \leq 1 / \rho, \quad r=1,2, \ldots, n,
$$

where

$$
\rho=\min \left(\alpha, p_{1} / p_{0}\right)
$$

provided that $\alpha \geq \mathrm{B}$ and $p_{1} / p_{0} \geq \mathrm{B}$. To arrive at a similar bound for $q_{r} / q_{r-1}$, we now define $q_{r}$ to be the solution of (5.1) that satisfies

$$
q_{n-1}=1, \quad q_{n}=0 .
$$

This solution can be generated by backward recurrence:

$$
c_{r} q_{r-1}=b_{r} q_{r}-a_{r} q_{r+1}, \quad r=n-1, n-2, \ldots, 1 .
$$

By applying Theorem 5.1 to this form of the difference equation, we deduce that

$$
q_{r} / q_{r-1} \leq \mathrm{B}, \quad r=1,2, \ldots, n .
$$

If we now restrict $\alpha>\mathrm{B}$ and $p_{1} / p_{0}>\mathrm{B}$, then $\rho>\mathrm{B}$, implying that $q_{r} / p_{r}$ is decreasing for $r=0,1, \ldots, n$. Accordingly, we may substitute in (5.4) and (5.10) by means of (5.16) and (5.18). This yields the required bounds in the form

$$
t_{0} \leq \frac{\mathrm{B}}{\rho-\mathrm{B}} ; \quad t_{j} \leq \frac{\rho}{\rho-\mathrm{B}}, \quad 1 \leq j \leq n .
$$

It is now easy to see how to compute a posteriori bounds for $\left|p_{r}-\bar{p}_{r}\right|$ successively for $r=2,3, \ldots, n$. Write

$$
T_{r}=t_{0} \frac{\left|\phi_{0}\right|}{p_{0}}+t_{1} \frac{\left|\phi_{1}\right|}{p_{1}}+\sum_{j=2}^{r} t_{j} \frac{\left|\phi_{j}\right|}{a_{j-1} p_{j}}, \quad r \geq 1,
$$

with the understanding that the term $t_{0}\left|\phi_{0}\right| / p_{0}$ is omitted in the case $p_{0}=\bar{p}_{0}=0$ and the empty sum is zero in the case $r=1$. From (5.12) we derive

$$
p_{r}-\bar{p}_{r}=\vartheta_{r} T_{r-1} p_{r}+\vartheta_{r} t_{r} \frac{\left|\phi_{r}\right|}{a_{r-1}}, \quad 2 \leq r \leq n,
$$

where $\vartheta_{r}$ is some number in the interval $[-1,1]$. Solving for $p_{r}$ we deduce that

$$
\left|p_{r}-\bar{p}_{r}\right| \leq \frac{1}{1-T_{r-1}}\left(T_{r-1} \bar{p}_{r}+t_{r} \frac{\left|\phi_{r}\right|}{a_{r-1}}\right)
$$

provided that $T_{r-1}<1$. As in Section 2 write $\varepsilon_{r}=p_{r}-\bar{p}_{r}$, and suppose that we have arrived at a lower bound for $p_{r-1}$ and upper bounds for $\left|\varepsilon_{r-1}\right|$ and $\left|T_{r-1}\right|$, with $r \geq 2$. Inequalities (5.19) and (5.21) immediately yield an upper bound for $\left|\varepsilon_{r}\right|$. A lower bound for $p_{r}$ can then be obtained, for example, from the inequality

$$
p_{r} \geq \bar{p}_{r}-\left|\varepsilon_{r}\right|
$$


(as long as $\left|\varepsilon_{r}\right|<\bar{p}_{r}$ ). And since

$$
T_{r}=T_{r-1}+\frac{t_{r}\left|\phi_{r}\right|}{a_{r-1} p_{r}}, \quad r \geq 2,
$$

we can also find an upper bound for $T_{r}$. The cycle is now ready to be repeated.

A more interesting problem is to extend the foregoing analysis to yield a priori bounds. As in Section 3, we suppose that the stored values of the coefficients $\bar{a}_{r}$, $\bar{b}_{r}$ and $\bar{c}_{r}$ are correct to $\operatorname{rp}(\delta)$, the initial values $\bar{p}_{0}$ and $\bar{p}_{1}$ are correct to $\operatorname{rp}(\varpi)$ and the computations are carried out in floating-point arithmetic with $\operatorname{wrp}(\gamma)$.

THEOREM 5.2. Let $p_{r}$ and $q_{r}$ be solutions of (5.1) such that $p_{0} \geq 0, p_{r}>0$ when $r>0, q_{r}>0$ when $0 \leq r \leq n-1, q_{n} \geq 0$, and $q_{r} / p_{r}$ is decreasing for $0 \leq r \leq n$. Assume also the conditions of the preceding paragraph, and let $\varpi_{0}=\varpi_{1}=\varpi$ and

$$
\begin{aligned}
& \varpi_{r}=2\left[\left(t_{0}+t_{1}\right) \varpi\right. \\
& \left.\quad+\sum_{j=2}^{r} t_{j}\left\{\delta+2 \gamma+\left(\frac{b_{j-1}}{a_{j-1}} \frac{p_{j-1}}{p_{j}}+\frac{c_{j-1}}{a_{j-1}} \frac{p_{j-2}}{p_{j}}\right)(\delta+\gamma)\right\}\right],
\end{aligned}
$$

$r \geq 2$, with $t_{j}$ defined by (5.4) and (5.10). ${ }^{6}$ Then

$$
p_{r} \simeq \bar{p}_{r} ; \quad \operatorname{rp}\left(\varpi_{r}\right)
$$

provided that $\varpi_{r} \leq \varsigma$, where $\varsigma=0.265 \ldots$ is the positive root of the equation

$$
-\ln \left(1-\frac{z e^{3 z / 2}}{2-z e^{z / 2}}\right)=z .
$$

Proof. We first need an upper bound for the error term $\phi_{j}$ in (5.2). Since each arithmetic operation is accompanied by an abbreviation error of $\operatorname{rp}(\gamma)$, we apply the rules of rp error analysis [12] to obtain

$$
\left|\phi_{j}\right| \leq\left\{a_{j-1} \bar{p}_{j}(\delta+2 \gamma)+\left(b_{j-1} \bar{p}_{j-1}+c_{j-1} \bar{p}_{j-2}\right)(\delta+\gamma)\right\} e^{\delta+2 \gamma}, \quad j \geq 2 .
$$

Next, on comparing (5.7) with the given conditions we have

$$
\left|\phi_{0}\right| \leq p_{0} \varpi e^{\varpi}, \quad\left|\phi_{1}\right| \leq p_{1} \varpi e^{\varpi} .
$$

Substituting in (5.12) by means of these inequalities, we derive

$$
\begin{aligned}
\frac{\left|p_{r}-\bar{p}_{r}\right|}{p_{r}} \leq\left[\left(t_{0}+t_{1}\right) \varpi\right. & +\sum_{j=2}^{r} t_{j}\left\{\frac{\bar{p}_{j}}{p_{j}}(\delta+2 \gamma)\right. \\
& \left.\left.+\left(\frac{b_{j-1}}{a_{j-1}} \frac{\bar{p}_{j-1}}{p_{j}}+\frac{c_{j-1}}{a_{j-1}} \frac{\bar{p}_{j-2}}{p_{j}}\right)(\delta+\gamma)\right\}\right] e^{\hat{\delta}+2 \gamma}
\end{aligned}
$$

where

$$
\hat{\delta}=\max (\varpi, \delta) .
$$

We shall establish (5.25) by induction. Suppose that

$$
p_{j} \simeq \bar{p}_{j} ; \quad \operatorname{rp}\left(\varpi_{j}\right), \quad j=0,1, \ldots, r-1,
$$

${ }^{6}$ Again, when $p_{0}=\bar{p}_{0}=0$ we set $t_{0}=0$. 
as is certainly the case when $r=1$ and 2 . If we extract the term $t_{r}\left(\bar{p}_{r} / p_{r}\right)(\delta+2 \gamma)$ from within the square brackets of the right member of (5.27) and express it in the form

$$
t_{r}\left(\frac{\bar{p}_{r}}{p_{r}}-1\right)(\delta+2 \gamma)+t_{r}(\delta+2 \gamma)
$$

then with the aid of (5.29) and the fact that each of $\varpi_{0}, \varpi_{1}, \ldots, \varpi_{r-1}$ is bounded by $\varpi_{r},{ }^{7}$ we see that

$$
\frac{\left|p_{r}-\bar{p}_{r}\right|}{p_{r}} \leq\left\{t_{r}(\delta+2 \gamma) \frac{\left|p_{r}-\bar{p}_{r}\right|}{p_{r}}+\frac{1}{2} \varpi_{r} e^{\varpi_{r}}\right\} e^{\hat{\delta}+2 \gamma}
$$

Next, from (5.24), (5.28) and the inequalities $t_{1}>1, t_{2}>1$ it is easily seen that $t_{r}(\delta+2 \gamma)$ and $\hat{\delta}+2 \gamma$ are both bounded by $\frac{1}{2} \varpi_{r}$ when $r \geq 2$. It follows that

$$
\frac{\left|p_{r}-\bar{p}_{r}\right|}{p_{r}} \leq \frac{1}{2} \varpi_{r} \frac{\left|p_{r}-\bar{p}_{r}\right|}{p_{r}} e^{\varpi_{r} / 2}+\frac{1}{2} \varpi_{r} e^{3 \varpi_{r} / 2}
$$

and hence that

$$
-\ln \left(1-\frac{\left|p_{r}-\bar{p}_{r}\right|}{p_{r}}\right) \leq-\ln \left(1-\frac{\varpi_{r} e^{3 \varpi_{r} / 2}}{2-\varpi_{r} e^{\varpi_{r} / 2}}\right) \leq \varpi_{r},
$$

the last step being a consequence of the assumption $\varpi_{r} \leq \varsigma$; compare (5.26). Thus (5.29) holds when $j=r$.

For the purpose of constructing a priori bounds, Theorem 5.2 possesses the essential feature that the error bound for $\bar{p}_{r}$ is expressed in terms of the true solution $p_{r}$ rather than the computed solution $\bar{p}_{r}$. With the notation of Theorem 5.1 , and the assumptions $\alpha>\mathrm{B}, p_{1} / p_{0}>\mathrm{B}$, the conditions of Theorem 5.2 on the solution $q_{r}$ are satisfied, and we may apply (5.16) and (5.19). From (5.1) we have

$$
\frac{b_{j-1} p_{j-1}}{a_{j-1} p_{j}}=1+\frac{c_{j-1} p_{j-2}}{a_{j-1} p_{j}}
$$

accordingly, (5.24) may be simplified into

$$
\varpi_{r}=2\left[\left(t_{0}+t_{1}\right) \varpi+\sum_{j=2}^{r} t_{j}\left\{2 \delta+3 \gamma+\frac{c_{j-1}}{a_{j-1}} \frac{p_{j-2}}{p_{j}}(2 \delta+2 \gamma)\right\}\right] .
$$

Then by making the indicated substitutions we arrive at

$$
\varpi_{r} \leq \frac{2}{\rho-\mathrm{B}}\left[(\mathrm{B}+\rho) \varpi+\rho \sum_{j=2}^{r}\left\{2 \delta+3 \gamma+\frac{c_{j-1}}{a_{j-1}} \frac{1}{\rho^{2}}(2 \delta+2 \gamma)\right\}\right] .
$$

If we now introduce the quantity

$$
C=\max _{j \in[1, n-1]}\left(c_{j} / a_{j}\right)
$$

\footnotetext{
7 This follows from the definition (5.24) and the inequality $t_{1}>1$.
} 
then we are led to the further simplification

$$
\varpi_{r} \leq \frac{2}{\rho-\mathrm{B}}\left[(\mathrm{B}+\rho) \varpi+(r-1)\left\{(2 \delta+3 \gamma) \rho+\frac{2 C}{\rho}(\delta+\gamma)\right\}\right] .
$$

Remarks. (a) The coefficient 2 outside the square brackets in the definition (5.24) of $\varpi_{r}$ is arbitrary, to some extent. In fact any constant in excess of unity could be used instead, provided that an appropriate change is made in the definition of $\zeta$.

(b) By referring to the analysis in this section leading up to (5.12), it is easy to relate the terms on the right-hand side of (5.24) to the various errors introduced during the computations. Thus, the terms $\left(t_{0}+t_{1}\right) \varpi$ are contributed by the inherent errors in $\bar{p}_{0}$ and $\bar{p}_{1}$. In $\sum_{j=2}^{r}$ the terms $t_{j}(\delta+2 \gamma)$ stem from the inherent error in $\bar{a}_{j-1}$ and the two errors introduced on abbreviating the difference $\overline{b_{j-1} p_{j-1}}-$ $\overline{c_{j-1} p_{j-2}}$ and the quotient $\overline{b_{j-1} p_{j-1}-c_{j-1} p_{j-2}} / \bar{a}_{j-1}$. The remaining terms in $\sum_{j=2}^{r}$ stem from the inherent errors in $\bar{b}_{j-1}$ and $\bar{c}_{j-1}$, and the errors made in abbreviating the products $\bar{b}_{j-1} \bar{p}_{j-1}$ and $\bar{c}_{j-1} \bar{p}_{j-2}$.

(c) The bound (5.31) grows linearly with $r$, which is a necessary condition for it to be realistic. Moreover, if the coefficients $a_{r}, b_{r}$ and $c_{r}$ in the original equation are constants and $p_{1} / p_{0} \geq \alpha$ (now the largest root of the characteristic equation), then $\rho=\alpha$ and the right-hand side of (5.31) becomes exactly twice the limiting value of the combined maximum effects of the inherent and abbreviation errors.

\section{Numerical Examples.}

Example 6.1. We compute the Legendre functions $P_{r}(x)$ and $Q_{r}(x)$ from the recurrence relation

$$
(r+1) p_{r+1}=(2 r+1) x p_{r}-r p_{r-1}
$$

with the initial values

$$
P_{0}(x)=1, \quad P_{1}(x)=x, \quad Q_{0}(x)=\frac{1}{2} \ln \frac{1+x}{1-x}, \quad Q_{1}(x)=\frac{x}{2} \ln \frac{1+x}{1-x}-1 .
$$

We take $x=0.95$, with the understanding that this value may be in error by as much as \pm 0.000001 , and use six-decimal floating-point arithmetic, with chopping, for the calculation of $p_{r} \equiv P_{r}(x)$ and $q_{r} \equiv Q_{r}(x)$. The computed values $\bar{p}_{r}$ and $\bar{q}_{r}$ are given for $r=0,1, \ldots, 16$ in the second and third columns of Table 6.1(i).

Error bounds have been computed from the formulae given in Section 2. It transpires, for example, that $w_{r}=1$, all $r$. Upper bounds $\left|\varepsilon_{r}\right|^{\mathcal{A}}$ and $\left|\eta_{r}\right|^{A}$ for the absolute errors $\left|\varepsilon_{r}\right|$ and $\left|\eta_{r}\right|$ in $\bar{p}_{r}$ and $\bar{q}_{r}$, respectively, appear in the fourth and fifth columns of Table 6.1(i). Some of the intermediate computations are shown in Table 6.1(ii). Here, and in subsequent examples, the superscript $A$ ("above") is again used to signify upper bounds of the designated quantities, whereas in the final column the superscript $B$ ("below") on $E_{r}$ indicates that entries in this column are lower bounds for $E_{r}$. These calculations were carried out by the methods of [12] using four-decimal floating-point arithmetic with chopping, except that in the cases $r=0$ and 1 the values of $\left|\psi_{r}\right|^{A}$ were found from Formulae (6.1) with the aid of high-precision values of the logarithmic function. 
TABLE 6.1(i)

Legendre functions $P_{r}(x)$ and $Q_{r}(x)$

\begin{tabular}{rcccccr}
$r$ & \multicolumn{1}{c}{$\bar{p}_{r}$} & $\bar{q}_{r}$ & $10^{6}\left|\varepsilon_{r}\right|^{A}$ & $10^{6}\left|\eta_{r}\right|^{A}$ & $10^{6}\left|\varepsilon_{r}\right|$ & $10^{6}\left|\eta_{r}\right|$ \\
& & & & & & \\
0 & 1 & 1.83178 & 0 & 11.09 & 0 & $11.0 \ldots$ \\
1 & 0.950000 & 0.740192 & 1 & 11.80 & 1 & $11.7 \ldots$ \\
2 & 0.853750 & 0.138880 & 83.19 & 84.93 & $2.8 \ldots$ & $15.3 \ldots$ \\
3 & 0.718436 & -0.273566 & 138.8 & 102.8 & $6.7 \ldots$ & $14.9 \ldots$ \\
4 & 0.554085 & -0.558962 & 291.3 & 178.2 & $12.7 \ldots$ & $11.4 \ldots$ \\
5 & 0.372736 & -0.736972 & 441.6 & 315.9 & $17.8 \ldots$ & $8.1 \ldots$ \\
6 & 0.187445 & -0.817756 & 500.4 & 437.7 & $20.6 \ldots$ & $6.6 \ldots$ \\
7 & 0.0112185 & -0.811052 & 451.9 & 422.6 & $21.4 \ldots$ & $22.9 \ldots$ \\
8 & -0.144030 & -0.729138 & 475.2 & 546.6 & $18.6 \ldots$ & $52.7 \ldots$ \\
9 & -0.268424 & -0.587454 & 489.5 & 748.1 & $12.4 \ldots$ & $84.1 \ldots$ \\
10 & -0.354878 & -0.404126 & 498.8 & 902.4 & $9.3 \ldots$ & $109.8 \ldots$ \\
11 & -0.399597 & -0.198888 & 467.3 & 913.2 & $9.7 \ldots$ & $123.9 \ldots$ \\
12 & -0.402295 & 0.00830666 & 359.4 & 784.8 & $12.4 \ldots$ & $125.1 \ldots$ \\
13 & -0.366103 & 0.198763 & 535.5 & 903.5 & $19.8 \ldots$ & $112.6 \ldots$ \\
14 & -0.297193 & 0.356448 & 716.4 & 970.4 & $25.9 \ldots$ & $88.9 \ldots$ \\
15 & -0.204148 & 0.469164 & 834.4 & 994.6 & $29.9 \ldots$ & $57.4 \ldots$ \\
16 & -0.0971412 & 0.529380 & 839.7 & 924.6 & $31.5 \ldots$ & $16.4 \ldots$
\end{tabular}

TABLE 6.1(ii)

Legendre functions (continued)

$\begin{array}{rccccccc}r & 10^{6}\left|\phi_{r}\right|^{A} & 10^{6}\left|\psi_{r}\right|^{A} & 10^{6}\left|A_{r}\right|^{A} & 10^{6}\left|B_{r}\right|^{A} & 10^{6}\left|C_{r}\right|^{A} & 10^{6}\left|D_{r}\right|^{A} & E_{r}^{B} \\ 0 & 0 & 11.09 & 0 & 0 & 10.63 & 8.289 & - \\ 1 & 1 & 11.80 & 1.014 & 1.856 & 22.74 & 30.29 & - \\ 2 & 103.3 & 69.77 & 100.5 & 79.39 & 90.26 & 83.06 & 0.9998 \\ 3 & 150.6 & 46.09 & 232.2 & 101.6 & 131.4 & 90.70 & 0.9997 \\ 4 & 174.2 & 89.07 & 362.3 & 151.2 & 198.0 & 116.6 & 0.9996 \\ 5 & 169.3 & 189.2 & 462.4 & 249.2 & 307.0 & 225.4 & 0.9994 \\ 6 & 135.1 & 294.4 & 519.9 & 353.5 & 422.6 & 448.5 & 0.9992 \\ 7 & 74.25 & 378.2 & 541.2 & 419.9 & 500.5 & 768.5 & 0.9990 \\ 8 & 40.38 & 426.1 & 549.1 & 458.9 & 512.4 & 1129 & 0.9990 \\ 9 & 100.3 & 427.5 & 571.3 & 539.4 & 582.1 & 1460 & 0.9988 \\ 10 & 189.9 & 377.7 & 631.0 & 660.1 & 693.1 & 1705 & 0.9986 \\ 11 & 269.5 & 278.0 & 736.8 & 779.9 & 802.8 & 1842 & 0.9983 \\ 12 & 326.3 & 140.9 & 879.3 & 856.8 & 871.1 & 1896 & 0.9982 \\ 13 & 351.8 & 81.42 & 1034 & 871.9 & 916.4 & 1922 & 0.9981 \\ 14 & 340.4 & 212.6 & 1174 & 952.9 & 1008 & 1991 & 0.9980 \\ 15 & 291.0 & 383.5 & 1277 & 1070 & 1137 & 2157 & 0.9977 \\ 16 & 206.6 & 524.8 & 1337 & 1183 & 1261 & 2436 & 0.9975\end{array}$

Each of the quantities $\left|A_{r}\right|^{\mathcal{A}},\left|B_{r}\right|^{\mathcal{A}},\left|C_{r}\right|^{\mathcal{A}}$ and $\left|D_{r}\right|^{\mathcal{A}}$ appearing in Eqs. (2.4) grows monotonically with $r$, and very roughly in a linear fashion. The final error bounds $\left|\varepsilon_{r}\right|^{A}$ and $\left|\eta_{r}\right|^{A}$ exhibit some of the oscillatory character of the solutions $p_{r}$ and $q_{r}$. The overall sizes of $\left|\varepsilon_{r}\right|^{A}$ and $\left|\eta_{r}\right|^{A}$ are linked directly to the sizes of the bounds $\left|\phi_{r}\right|^{\mathcal{A}}$ and $\left|\psi_{r}\right|^{\mathcal{A}}$ for the abbreviation errors $\phi_{r}$ and $\psi_{r}$ in Eqs. (2.2).

Because of the uncertainty in the assumed value of $x$, the actual errors $\varepsilon_{r}$ and $\eta_{r}$ in $\bar{p}_{r}$ and $\bar{q}_{r}$ are unknown. However, their maximum absolute values can be found by taking $x=0.95 \pm 0.000001$ in turn, and recalculating $p_{r}$ and $q_{r}$ using 
higher precision. The results are shown in the last two columns of Table 6.1(i). Of course, the bounds $\left|\varepsilon_{r}\right|^{\mathcal{A}}$ and $\left|\eta_{r}\right|^{\mathcal{A}}$ overestimate the actual values of $\left|\varepsilon_{r}\right|$ and $\left|\eta_{r}\right|$ considerably. This is caused partly by the stochastic nature of the actual abbreviation errors, and partly by the "radix effect". Had the computations been carried out in base 2, for example, instead of base 10, then the overestimation of the actual errors would be reduced by a factor of about 2 or 3 [12], [17].

Example 6.2. Let us solve the system (1.2) of Example 1.1 by the first method of Section 4, that is, by using the recurrence relation satisfied by the ratios $h_{r} \equiv$ $p_{r} / p_{r-1}$. However, instead of assuming that the coefficients $a_{r}, b_{r}$ and $c_{r}$ in Eq. (4.1) are exactly 12, 25 and 13, respectively, we suppose that they are given in interval form $a_{r}=a, b_{r}=b, c_{r}=c$, all $r$, where

$$
a=[11.9999,12.0001], \quad b=[24.9998,25.0002], \quad c=[12.9999,13.0001] .
$$

The initial values $p_{0}=1, p_{1}=13 / 12$, are unchanged. (Of course, the method used in Example 1.1 would be just as unsuccessful with this modification.)

The recurrence formulae are given by

$$
a h_{r}=b-\left(c / h_{r-1}\right), \quad p_{r}=h_{r} p_{r-1}, \quad r \geq 2 .
$$

Interval values of $p_{r}$ and $h_{r}$, computed with six-figure decimal arithmetic, are given in Table 6.2. These results obviously represent a considerable improvement on those found on Table 1.1. However, they are not entirely satisfactory for the following reason. The interval width $I h_{r}$ of $h_{r}$ grows roughly in proportion to $r$ : this can be seen from the entries in the penultimate column of Table 6.2. This linear growth in I $h_{r}$ leads to an almost quadratic rate of growth in the corresponding relative errors of the $p_{r}$. This phenomenon is illustrated by the values of $I p_{r} /\left(r^{2} \bar{p}_{r}\right)$ supplied in the last column of Table 6.2 ; here $\bar{p}_{r}$ denotes the midpoint of the interval value of $p_{r}$.

TABLE 6.2

Interval solution of Eqs. (6.2)

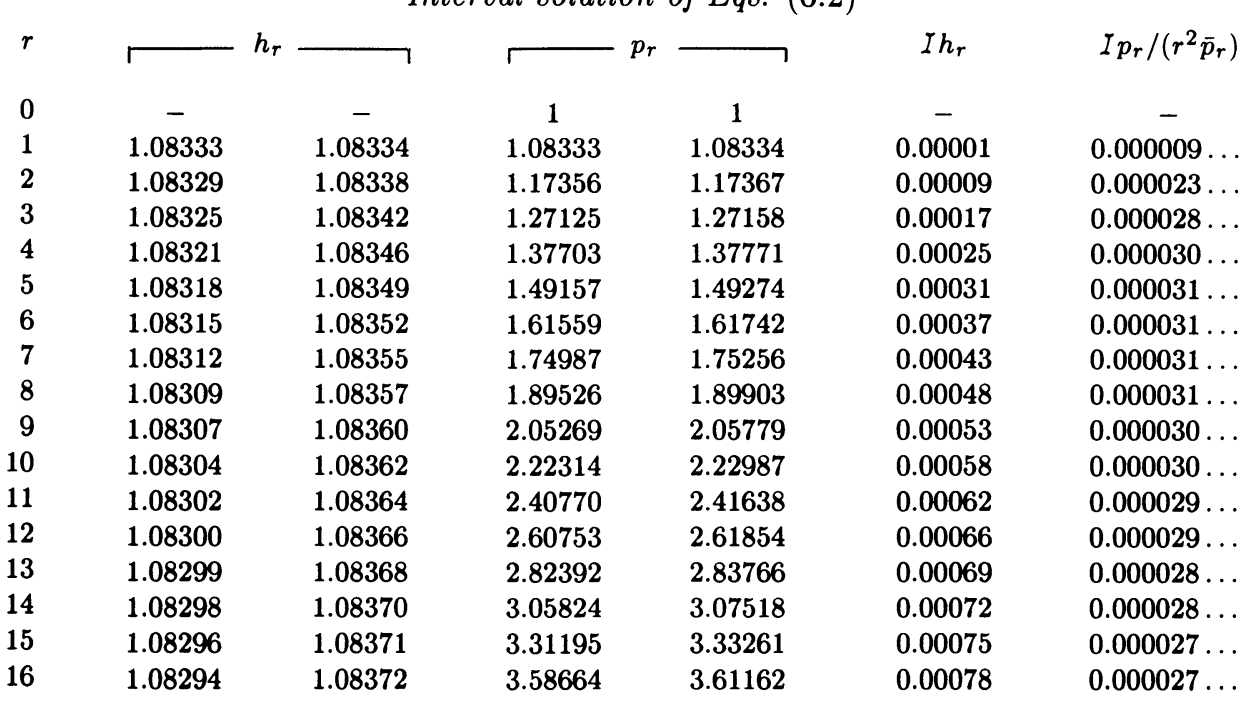

Example 6.3. We solve the problem posed in Example 6.2 by the second method of Section 4. On omitting the suffix $r$ from the coefficients $a_{r}, b_{r}, c_{r}, \lambda_{r}, \mu_{r}$ and 
$\nu_{r}$, we obtain the recurrence relations

$$
a u_{r}=\nu p_{r}+\mu u_{r-1}, \quad p_{r+1}=u_{r}+\lambda p_{r}, \quad r \geq 1,
$$

in which

$$
\lambda=b /(2 a), \quad \mu=c / \lambda, \quad \nu=b-a \lambda-\mu .
$$

The initial member of the sequence $\left\{u_{r}\right\}$ is given by $u_{0}=p_{1}-\lambda p_{0}$. Interval values of $\lambda, \mu$ and $\nu$ are found to be

$$
\lambda=[1.04164,1.04169], \quad \mu=[12.4797,12.4803], \quad \nu=[0.0196000,0.0204000],
$$

and using six-figure decimal arithmetic we arrive at the interval values of $p_{r}$ and $u_{r}$ displayed in Table 6.3.

For large $r$, the intervals containing $p_{r}$ are narrower than those obtained in Table 6.2 but from the last column, in which $\bar{p}_{r}$ again denotes the mean value of $p_{r}$, it is evident that the growth of the relative error is still not linear in $r$.

TABLE 6.3

Interval solution of Eqs. (6.3)

\begin{tabular}{rccccc}
$r$ & \multicolumn{3}{c}{$u_{r}$} & \multicolumn{3}{c}{$p_{r}$} & $I p_{r} /\left(r \bar{p}_{r}\right)$ \\
& $\Gamma$ & & & & \\
1 & 0.0416400 & 0.0417000 & 1 & 1 & - \\
2 & 0.0450735 & 0.0452113 & 1.08333 & 1.08334 & $0.00000 \ldots$ \\
3 & 0.0487915 & 0.0490168 & 1.17350 & 1.17373 & $0.00009 \ldots$ \\
4 & 0.0528176 & 0.0531412 & 1.27115 & 1.27169 & $0.00014 \ldots$ \\
5 & 0.0571773 & 0.0576112 & 1.37689 & 1.37786 & $0.00017 \ldots$ \\
6 & 0.0618983 & 0.0624557 & 1.49139 & 1.49293 & $0.00020 \ldots$ \\
7 & 0.0725463 & 0.0733965 & 1.74965 & 1.75279 & $0.00025 \ldots$ \\
8 & 0.0785408 & 0.0795638 & 1.89504 & 1.89927 & $0.00027 \ldots$ \\
9 & 0.0850317 & 0.0862483 & 2.05248 & 2.05803 & $0.00030 \ldots$ \\
10 & 0.0920608 & 0.0934933 & 2.22297 & 2.23008 & $0.00031 \ldots$ \\
11 & 0.0996716 & 0.101346 & 2.40759 & 2.41656 & $0.00033 \ldots$ \\
12 & 0.107913 & 0.109856 & 2.60751 & 2.61866 & $0.00035 \ldots$ \\
13 & 0.116838 & 0.119079 & 2.82399 & 2.83770 & $0.00037 \ldots$ \\
14 & 0.126502 & 0.129076 & 3.05841 & 3.07509 & $0.00038 \ldots$ \\
15 & 0.136967 & 0.139910 & 3.31226 & 3.33238 & $0.00040 \ldots$ \\
16 & - & - & 3.58714 & 3.61122 & $0.00041 \ldots$
\end{tabular}

Example 6.4. We compute the absolute value of the Bessel function $Y_{r}(x)$ by forward recurrence from the relation

$$
p_{r+1}=(2 r / x) p_{r}-p_{r-1}
$$

We take $x=100$ and the initial values

$$
p_{100}=-Y_{100}(100)=0.166921 \ldots, \quad p_{101}=-Y_{101}(100)=0.200285 \ldots .
$$

Using six-decimal floating-point arithmetic, with chopping, we obtain the values $\bar{p}_{r}$ given in the second column of Table 6.4.

We shall compute both a posteriori and a priori error bounds by the methods of Section 5. These computations are carried out in four-decimal floating-point arithmetic with chopping. In the terminology of [12] this is the lower mode of computation $(\mathcal{L})$, and its associated wrp is $\gamma_{\ell}=10^{-3}$. For the computation of the $\bar{p}_{r}$, the wrp is $\gamma=10^{-5}$. 
Both types of error bound require the evaluation of the bounds (5.19) for the coefficients $t_{j}$. The zeros of the local characteristic polynomial $z^{2}-(2 r / x) z+1$ are given by

$$
\alpha_{r}=(r / x)+\left\{(r / x)^{2}-1\right\}^{1 / 2}, \quad \beta_{r}=(r / x)-\left\{(r / x)^{2}-1\right\}^{1 / 2} .
$$

Consequently, for any $n$ exceeding 100 , we have

$$
\alpha=\alpha_{101}=1.15177 \ldots, \quad \mathrm{B}=\beta_{101}=0.868225 \ldots ;
$$

compare (5.13). Also, from (5.17) and (6.5) we see that $\rho=\alpha$. From (5.19) we derive

$$
t_{100} \leq 3.061 \ldots ; \quad t_{j} \leq 4.061 \ldots, \quad j \geq 101 .
$$

For simplicity, however, we use the same bound for all $j$ :

$$
t_{j}<4.062, \quad j \geq 100 .
$$

For a posteriori error bounds we need to compute bounds for the quantities $\phi_{r}$ defined by

$$
\bar{p}_{r}=\{(2 r-2) / x\} \bar{p}_{r-1}-\bar{p}_{r-2}+\phi_{r}, \quad r \geq 102
$$

compare (5.2). Since the coefficient $(2 r-2) / x$ is exact, only two chopping errors are introduced at each recurrence step. Applying the methods of [12] we find that

$$
\left|\phi_{r}\right| \leq \overline{\bar{\chi}}_{r} \gamma e^{3 \gamma_{\ell}}, \quad r \geq 102,
$$

where

$$
\chi_{r}=p_{r}+\{(2 r-2) / x\} p_{r-1},
$$

\begin{tabular}{|c|c|c|c|c|c|c|c|}
\hline \multicolumn{8}{|c|}{ Bessel function $-Y_{r}(x)$} \\
\hline $\bar{p}_{r}$ & $\overline{\bar{\chi}}_{r}$ & $10^{5} T_{r}^{A}$ & $p_{r}^{B}$ & $10^{5}\left|\varepsilon_{r}\right|^{A}$ & $\left|\varepsilon_{r} / \bar{p}_{r}\right|^{\AA}$ & $\varepsilon_{r} / \bar{p}_{r}$ & $\varpi_{r}^{A}$ \\
\hline 0.166921 & - & - & - & - & - & - & - \\
\hline 0.200285 & - & 4.531 & - & - & - & - & - \\
\hline 0.237654 & 0.6421 & 15.70 & 0.2375 & 3.755 & 0.00016 & $0.000004 \ldots$ & 0.00037 \\
\hline 0.284529 & 0.7693 & 26.94 & 0.2844 & 7.710 & 0.00028 & $0.000007 \ldots$ & 0.00060 \\
\hline 0.348475 & 0.9345 & 38.14 & 0.3482 & 13.35 & 0.00039 & $0.000011 \ldots$ & 0.00082 \\
\hline 0.440299 & 1.165 & 49.20 & 0.4399 & 21.78 & 0.00050 & $0.000013 \ldots$ & 0.00104 \\
\hline 0.576152 & 1.500 & 60.13 & 0.5757 & 34.86 & 0.00061 & $0.000016 \ldots$ & 0.00127 \\
\hline 0.781141 & 2.002 & 70.94 & 0.7805 & 55.76 & 0.00072 & $0.000021 \ldots$ & 0.00149 \\
\hline 1.09548 & 2.766 & 81.61 & 1.094 & 89.94 & 0.00083 & $0.000032 \ldots$ & 0.00172 \\
\hline 1.58508 & 3.951 & 92.21 & 1.583 & 147.0 & 0.00093 & $0.000048 \ldots$ & 0.00194 \\
\hline 2.35999 & 5.814 & 102.7 & 2.356 & 243.8 & 0.00104 & $0.000057 \ldots$ & 0.00216 \\
\hline 3.60689 & 8.797 & 113.1 & 3.601 & 410.8 & 0.00114 & $0.000063 \ldots$ & 0.00239 \\
\hline 5.64730 & 13.65 & 123.5 & 5.639 & 702.6 & 0.00125 & $0.000067 \ldots$ & 0.00261 \\
\hline 9.04301 & 21.68 & 133.9 & 9.030 & 1218 & 0.00135 & $0.000074 \ldots$ & 0.00284 \\
\hline 14.7899 & 35.21 & 144.3 & 14.75 & 2147 & 0.00146 & $0.000077 \ldots$ & 0.00306 \\
\hline 24.6778 & 58.39 & 154.6 & 24.63 & 3840 & 0.00156 & $0.000084 \ldots$ & 0.00328 \\
\hline 41.9690 & 98.71 & 164.8 & 41.89 & 6966 & 0.00166 & $0.000088 \ldots$ & 0.00351 \\
\hline 72.6902 & 170.0 & 175.1 & 72.56 & 12810 & 0.00177 & $0.000090 \ldots$ & 0.00373 \\
\hline 128.126 & 298.1 & 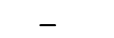 & - & 23920 & 0.00187 & $0.000092 \ldots$ & 0.00396 \\
\hline
\end{tabular}

and the double bar signifies the value computed in $\mathcal{L}$. The rest of the computation proceeds in accordance with the relations (5.21), (5.22) and (5.23), as described in Section 5. The main steps are shown in columns 3, 4, 5 and 6 of Table 6.4: As in Example 6.1, the superscripts $A$ and $B$ signify upper and lower bounds respectively. Again, these bounds were computed using the methods of [12].

TABLE 6.4 
By way of comparison, the seventh column of Table 6.4 gives an upper bound $\left|\varepsilon_{r} / \bar{p}_{r}\right|^{2}$ for the relative error. This is derived from the entries in the second and sixth columns. The next column gives the value of the actual relative error $\varepsilon_{r} / \bar{p}_{r}$ computed by use of high-precision values of $Y_{r}(100)$. Our bound overestimates the true error by a factor that ranges from about 35 at the beginning of the recurrences down to about 20 at $r=118$. Two sources contribute to this factor. First, there is the radix effect associated with base 10. As we observed in Example 6.1, use of base 2 instead might save a factor of about 2 or 3 . Secondly, we have used a uniform bound, given by (6.7), for the $t_{j}$. In fact, most of these coefficients are considerably less than 4.062. If desired, smaller bounds could be used without changing the $O(r)$ estimate of the total computing effort. For example, since the sequence $\beta_{r}$ is decreasing, it is easy to see that the second of the bounds (5.19) can be replaced by

$$
t_{j} \leq \alpha /\left(\alpha-\beta_{j}\right), \quad 101 \leq j \leq n .
$$

The quantity $\alpha /\left(\alpha-\beta_{j}\right)$ has the values $2.258 \ldots$ and $1.925 \ldots$ at $j=110$ and 118 , respectively. Further sharpening is possible by application of the theorems given in [6, Section 5].

The final column of Table 6.4 gives a priori bounds $\varpi_{r}^{A}$ for the relative precision of the approximation $\bar{p}_{r}$ to $p_{r}$. These were found as follows. Since the coefficients in (6.4) are exact, we have $\delta=0$. Also, since $c_{j-1}=a_{j-1}$, all $j$, and only two chopping errors are made at each recurrence step, Eq. (5.24) may be replaced by

$$
\varpi_{r}=2\left\{\left(t_{100}+t_{101}\right) \varpi+\gamma \sum_{j=102}^{r} t_{j}\left(2+\frac{p_{j-2}}{p_{j}}\right)\right\}, \quad j \geq 102 .
$$

On taking $\varpi=\gamma=10^{-5}$, substituting for the $t_{j}$ by means of (6.6) and using the fact that $p_{j-2} / p_{j} \leq 1 / \rho^{2}$, all $j$, we arrive at the numerical form

$$
\varpi_{r} \leq\{14.25+(22.40)(r-101)\} \times 10^{-5}, \quad r \geq 102 .
$$

As expected, the values of $\varpi_{r}^{A}$ are approximately twice the size of the a posteriori relative error bounds $\left|\varepsilon_{r} / \bar{p}_{r}\right|^{\mathcal{A}}$.

7. Conclusions. We have described various methods for computing error bounds for solutions of difference equations of the form

$$
a_{r} p_{r+1}=b_{r} p_{r}+c_{r} p_{r-1}
$$

that are generated by forward recurrence. Two cases are considered: (A) oscillatory systems, in which $b_{r}^{2}+4 a_{r} c_{r}<0$, all $r$; (B) monotonic systems, in which $b_{r}^{2}+4 a_{r} c_{r} \geq$ 0 , all $r$. In Case $\mathrm{B}$ methods have been provided for finding bounds of both a posteriori and a priori types. In Case A, only an a posteriori method is available, and there is a need for a method for constructing a priori bounds analogous to that of Section 5.

Acknowledgments. Helpful comments on this work have been made by J. R. Cash, E. Kaucher and D. W. Lozier, and especially the referee. I am also pleased to acknowledge the assistance of Dr. Lozier with the generation of high-precision values of the Legendre and Bessel functions needed in Examples 6.1 and 6.4. 
University of Maryland

Institute for Physical Science and Technology

College Park, Maryland 20742

National Bureau of Standards

Mathematical Analysis Division

Gaithersburg, Maryland 20899

1. J. R. CASH, Stable Recursions, Academic Press, London, 1979.

2. W. GAUTSCHI, "Computational aspects of three-term recurrence relations," SIAM Rev., v. 9, 1967, pp. 24-82.

3. W. GaUtSCHI, "Zur Numerik rekurrenter Relationen," Computing, v. 9, 1972, pp. 107126. [Translated as Report ARL 73-0005, Aerospace Research Laboratories, Wright-Patterson Air Force Base, Ohio, 1973.]

4. W. GautsCHI, "Computational methods in special functions-a survey," in Theory and Application of Special Functions (R. A. Askey, ed.), Academic Press, New York, 1975, pp. 1-98.

5. N. J. HIGHAM, "Efficient algorithms for computing the condition number of a tridiagonal matrix," SIAM J. Sci. Statist. Comput., v. 7, 1986, pp. 150-165.

6. R. M. M. MAтTHEIJ, "Accurate estimates of solutions of second order recursions," Linear Algebra Appl., v. 12, 1975, pp. 29-54.

7. R. M. M. MAtTheiJ \& A. VAN DER Sluis, "Error estimates for Miller's algorithm," Numer. Math., v. 26, 1976, pp. 61-78.

8. R. E. MOORE, Methods and Applications of Interval Analysis, Society for Industrial and Applied Mathematics, Philadelphia, 1979.

9. F. W. J. OLvER, "Error analysis of Miller's recurrence algorithm," Math. Comp., v. 18, 1964, pp. 65-74.

10. F. W. J. OLVER, "Numerical solution of second-order linear difference equations," J. Res. Nat. Bur. Standards Sect. B, v. 71, 1967, pp. 111-129.

11. F. W. J. OlVER, "Bounds for the solutions of second-order linear difference equations," $J$.

Res. Nat. Bur. Standards Sect. B, v. 71, 1967, pp. 161-166.

12. F. W. J. OlvER, "Further developments of rp and ap error analysis, "IMA J. Numer. Anal., v. 2, 1982, pp. 249-274.

13. F. W. J. Olver \& J. H. Wilkinson, "A posteriori error bounds for Gaussian elimination," IMA J. Numer. Anal., v. 2, 1982, pp. 377-406.

14. S. M. RUMP, "Solving algebraic problems with high accuracy," in A New Approach to Scientific Computation (U. W. Kulisch and W. L. Miranker, eds.), Academic Press, New York, 1983, pp. 51-120.

15. A. VAN DER SLUIS, "Estimating the solutions of slowly varying recursions," SIAM J. Math. Anal., v. 7, 1976, pp. 662-695.

16. R. TAIT, "Error analysis of recurrence relations," Math. Comp., v. 21, 1967, pp. 629-638.

17. P. R. TURNER, "The distribution of leading significant digits," IMA J. Numer. Anal., v. 2, 1982, pp. 407-412.

18. J. H. Wilkinson, Rounding Errors in Algebraic Processes, National Physical Laboratory Notes on Applied Science No. 32, Her Majesty's Stationery Office, London, 1963.

19. J. WIMP, Computation with Recurrence Relations, Pitman, Boston, 1984. 